\title{
MRI Evaluation of Cervical Carcinoma Stage II at a Tertiary Care Hospital in Dhaka City
}

\author{
Rokshana Ahmed ${ }^{1}$, Shaifur Ahmed², Md. Abdul Alim³, Zerin Haque \\ Noor-E-Ferdous ${ }^{5}$, Mst. Nazmun Nahar ${ }^{6}$
}

\begin{abstract}
${ }^{1}$ Assistant Professor, Department of Neuro-radiology and Imaging, National Institute of Neurosciences and Hospital, Dhaka, Bangladesh; ${ }^{2}$ Medical Officer, Department of Physical Medicine \& Neurorehabilitation, National Institute of Neurosciences and Hospital, Bangladesh; ${ }^{3}$ Assistant Professor, Department of Neuro-radiology and Imaging, National Institute of Neurosciences and Hospital, Bangladesh; ${ }^{4} J u n i o r$ Consultant, Department of Neuro-radiology and Imaging, National Institute of Neurosciences and Hospital, Dhaka, Bangladesh; ${ }^{5}$ Assistant Professor, Department of Gynaecological Oncology, Banghabandhu Sheikh Mujib Medical University, Dhaka, Bangladesh; ${ }^{6}$ Associate Professor \& Head, Department of Neuro-radiology and Imaging, National Institute of Neurosciences and Hospital, Dhaka, Bangladesh
\end{abstract}

[Received on: 22 April 2021; Accepted on: 12 May 2021; Published: 1 July 2021]

\begin{abstract}
Background: Diagnosis of clinical staging is very crucial for the management of cervical carcinoma. Objective: The purpose of the present study was to evaluate the MRI and clinical staging of IIA and IIB cervical carcinoma. Methodology: This cross-sectional study was conducted in Department of Radiology \& Imaging at Dhaka Medical College Hospital, Dhaka, Bangladesh with the collaboration of Obstetrics and Gynecology unit as well as the Department of Pathology of Dhaka Medical College, Dhaka, Bangladesh from July 2010 to June 2012 for a period of two years. All patients with MRI examination who had given the written permission for the use of their data for research purposes at the time of their scan were selected as study population. The cervix and vagina were visually inspected for superficial evidence of the extent of tumor. Biopsied were not routinely performed since patients were usually referred only after a biopsy and had confirmed the diagnosis of invasive carcinoma. Assessments of the extent of tumor, parametrial extension, lymph nodes were evaluated. Then the findings of MRI clinical examination, surgical findings and histopathological reports were recorded on a preformed data sheet. Finally, the diagnostic effectiveness of MRI staging and clinical FIGO staging were evaluated against the surgico-histopathological reports. Result: A total number of 30 patients were recruited for this study. The mean age with SD of the study population were $57.82 \pm 13.34$ years. The sensitivity, specificity, PPV, NPV and accuracy of MRI of the detection of IIA of cervical carcinoma was $80.0 \%, 100.0 \%, 100.0 \%, 90.9 \%$ and $93.33 \%$ respectively. However, the sensitivity, specificity, PPV, NPV and accuracy of clinical examination of the detection of IIA of cervical carcinoma was $84.34 \%, 63.86 \%, 84.87 \%, 45.86 \%$ and $67.44 \%$ respectively. Conclusion: In conclusion the diagnostic validity of MRI for the detection of carcinoma cervix stage IIA is better than clinical examination. [Journal of National Institute of Neurosciences Bangladesh, July 2021;7(2):113-117]
\end{abstract}

Keywords: Diagnostic evaluation; MRI; clinical staging; cervical carcinoma

Correspondence: Dr. Rokshana Ahmed, Assistant Professor, Department of Radiology and Imaging, National Institute of Neurosciences and Hospital, Dhaka, Bangladesh; Email: rokshanaahmed192@gmail.com; Cell no.: +8801715400684

Conflict of interest: There is no conflict of interest relevant to this paper to disclose.

Funding agency: This research project was not funded by any group or any institution.

Contribution to authors: Ahmed R, Ahmed S, Alim A, Nahar MN were involved in protocol preparation, data collection and literature search and manuscript writing. Haque Z, Ferdous NE were involved in preparation and revision of this manuscript.

How to cite this article: Ahmed R, Ahmed S, Alim MA, Haque Z, Ferdous NE, Nahar MN. MRI Evaluation of Cervical Carcinoma Stage II at a Tertiary Care Hospital in Dhaka City. J Natl Inst Neurosci Bangladesh, 2021;7(2): 113-117

Copyright: (C2021. Ahmed et al. Published by Journal of National Institute of Neurosciences Bangladesh. This article is published under the Creative Commons CC BY-NC License (https://creativecommons.org/licenses/by-nc/4.0/). This license permits use, distribution and reproduction in any medium, provided the original work is properly cited, and is not used for commercial purposes.

\section{Introduction}

Cervical cancer is the only gynecologic cancer still clinically staged ${ }^{1}$. Staging is based on clinical FIGO criteria. Worldwide, it is second most common and the fifth deadliest cancer in women ${ }^{2}$. It affects about 16 per 100,000 women per year and kills about 9 per 100,000 per year; however, approximately $80.0 \%$ of cervical cancers occur in developing countries ${ }^{3}$. Worldwide, it 
was estimated that there were 473,000 cases of cervical cancer and 253,500 deaths per year ${ }^{4}$.

Reports from the tertiary level hospitals show that incidence of cervical cancer is increasing in our country also. Invasive cervical carcinoma, the third most common malignancy of the reproductive tract of women under age of 50 years, have five-year survival rates vary between $92.0 \%$ for stage I disease and $17.0 \%$ for stage IV disease ${ }^{5}$. No reliable statistical data about cancer are available for most developing countries in general and Bangladesh, in particular. In the light of statistics available from WHO cancer incidence and prevalence can be estimated approximately as 200000 and 800000 respectively for 130 million people of Bangladesh ${ }^{6}$. According to the National Institute of Cancer Research and Hospital (NICRH), Dhaka, cancer registry report that covers three years, cervical cancer is the second most common cancers among females and ranked third among the whole group ${ }^{7}$. On the other hand, it was the most common cancer among female group based on the data available from Radiotherapy Department of Dhaka Medical College and Hospital.

The greatest difficulties in the clinical evaluation of patients with cervical cancer are the estimation of tumor size, especially when the tumor is endo-cervical in location; the assessment of parametrial and pelvic sidewall invasion and the evaluation of lymph node and distant metastases ${ }^{8}$. MRI is the best single imaging investigation that can accurately determine tumor volume, location, depth of stromal invasion and extension into lower uterine and vaginal segments. It is accurate for evaluation of tumor size, usually within 0.5 $\mathrm{cm}$ of the surgical size, in $70.0 \%$ to $90.0 \%$ cases $^{9}$. It is also useful in the evaluation of lymph node metastases. Amongst these all various prognostic factors tumor size has emerged as the most important factor to which other morphological risk factors like tumor invasion depth, loco-regional extent and lymph node involvement are related $^{10}$.

MRI is the modern diagnostic procedure and it has shown advantages over existent techniques in imaging of female pelvis ${ }^{7}$. It has complemented sonography and MRI or CT-scan in further refining anatomic details and allowing the display of the disease in more detail. Sonography remains the screening technique for the many uterine and ovarian afflictions. However, its significant operator dependence, limitations due to patients' habitus and relative inability to tissue characterization, significantly decrease its value ${ }^{11}$. CT-scan which is currently used for the staging of pelvic neoplasm, also has limitations. These include the presence of ionizing radiation and distortion due to metallic clips, contrast media, and bone density. All this restricts the usefulness of pelvic CT-scan, particularly in regard to soft tissue resolution ${ }^{12}$. The advantages of MRI in the study of pelvic abnormalities have recently been described. MRI is non-invasive, does not depend on ionization, has superb soft tissue contrast resolution, and is capable of multidirectional imaging, simultaneous imaging of multiple sections, and visualization of blood vessels without the need of contrast injection ${ }^{13}$. The purpose of the present study was to evaluate the MRI and clinical staging of IIA and IIB cervical carcinoma.

\section{Methodology}

This analytical cross-sectional study was conducted in Department of Radiology \& Imaging at Dhaka Medical College Hospital, Dhaka, Bangladesh with the collaboration of Obstetrics and Gynecology unit as well as the Department of Pathology of Dhaka Medical College, Dhaka, Bangladesh from July 2010 to June 2012 for a period of two years. The study protocol was approved by Ethical Review Committee of Dhaka Medical College. All patients with MRI examination who had given the written permission for the use of their data for research purposes at the time of their scan were selected as study population. The patients with biopsy confirmed invasive cervical carcinoma of uterine cervix were evaluated with MR imaging were scheduled for surgery based on clinical assessment. Exclusion criteria were patients not suitable for surgery or refused to do MRI scan and surgery, patients in whom surgicohistopathological report were not available. Patients were examined under anesthesia by the gynecologic surgeon to determine the appropriate clinical FIGO stage. The cervix and vagina were visually inspected for superficial evidence of the extent of tumor. Biopsied were not routinely performed since patients were usually referred only after a biopsy and had confirmed the diagnosis of invasive carcinoma. However, if vaginal lesions were identified that would change the clinical stage, these were biopsied at the time of examination under anesthesia. Bimanual palpation was performed to assess the bulk of the cervix and to estimate the tumor volume. The parametria, utero-sacral ligaments, and pelvic side wars were palpated to assess tumor extension. The rectum was examined manually and sigmoidoscopically to check for tumor extension. Cystoscopy was also performed to detect bladder involvement. Last of all the attending gynecologist assigned the clinical stage for each patient according to FIGO criteria. MRI examination were 
performed in all patients by using 0.3 Tesla, Hitachi-MRI machine. Patients were instructed to fast for 4-6 hours before the MRI examination to limit artifact due to small bowel peristalsis. In each patient, images were obtained with $1 \mathrm{~cm}$ thick contiguous sections in transverse, sagittal and coronal planes. The matrix was $256 \times 256$, with a $32 \mathrm{~cm}$ field of view, and the data were displayed in a $512 \times 512$ matrix. Imaging protocols was preset in machine. Post contrast images were achieved by delivering a bolus $(50 \mathrm{ml})$ Gadolinium DTPA in intravenous route manually by medium hand pressure (at a rate of about 1 to $1.5 \mathrm{ml} / \mathrm{sec}$ with 19/20-gauge needle) and completed by 50 to 60 sec. All MRI findings were interpreted without knowledge of the clinical or surgicohistopathological findings and based on specific MR imaging staging criteria by the investigator and one of the expert radiologists of the department. Assessments of the extent of tumor, parametrial extension, lymph nodes were evaluated. Then the findings of MRI clinical examination, surgical findings and histopathological reports were recorded on a preformed data sheet. Finally, the diagnostic effectiveness of MRI staging and clinical FIGO staging were evaluated against the surgico-histopathological reports. Analyses were performed with SPSS software, versions 22.0 (IBM SPSS Statistics for Windows, Version 22.0. Armonk, NY: IBM Corp.). Continuous data that were normally distributed were summarized in terms of the mean, standard deviation, median, minimum, maximum and number of observations. Skewed data were presented in terms of the maximum, upper quartile, median, lower quartile, minimum and number of observations. Categorical or discrete data were summarized in terms of frequency counts and percentages. Sensitivity, specificity and positive and negative predictive values (PPV and NPV) were calculated by comparing the results MRI staging and clinical FIGO staging with surgico-histopathological results. Where appropriate, results were reported with $95 \%$ confidence intervals.

\section{Results}

A total number of 30 patients were recruited for this study. Most of the study population were in the age group of 40 to 44 Years which was 11(36.7\%) cases followed by 45 to 49 years, 50 to 54 years and 55 to 59 years which were $5(16.7 \%)$ cases, $4(13.3 \%)$ cases, $4(13.3 \%)$ cases respectively. However, both 35 to 39 years and 60 years and above had $3(10.0 \%)$ cases in each. The mean age with SD of the study population were $57.82 \pm 13.34$ years with the range of 35 to 70 years (Table 1).

Table 1: Age Distribution of the Study Subjects

\begin{tabular}{lcc}
\hline Age Group & Frequency & Percent \\
\hline 35 to 39 Years & 3 & 10.0 \\
40 to 44 Years & 11 & 36.7 \\
45 to 49 Years & 5 & 16.7 \\
50 to 54 Years & 4 & 13.3 \\
55 to 59 Years & 4 & 13.3 \\
60 Years and above & 3 & 10.0 \\
Total & $\mathbf{3 0}$ & $\mathbf{1 0 0 . 0}$ \\
Mean \pm SD (Years) & $57.82 \pm 13.34(35$ to 70) \\
\hline
\end{tabular}

MRI staging of cervical cancer of IIA and IIB were compared with the surgical stage of IIA and IIB. It was found that 8 cases of patients were confirmed by both clinical and surgical staging as IIA. Again, 4 cases were confirmed negative of IIB stage by both clinical stage as well as surgical staging. The difference between the MRI staging and surgical staging for the detection of cervical carcinoma of stage IIA and IIB were not statistically significant $(\mathrm{p}=0.001)$ (Table 2$)$.

Table 2: MR imaging Stage Compared with Surgical Stage

\begin{tabular}{lcccc}
\hline \multirow{2}{*}{$\begin{array}{l}\text { Surgical } \\
\text { Stage }\end{array}$} & \multicolumn{2}{c}{ MRI Findings } & Total & P value \\
\cline { 2 - 3 } & IIA & IIB & & \\
\hline IIA & 8 & 1 & 9 & \\
IIB & 1 & 4 & 5 & \\
Total & $\mathbf{9}$ & $\mathbf{5}$ & $\mathbf{1 4}$ & \\
\hline
\end{tabular}

The sensitivity, specificity, PPV, NPV and accuracy of MRI of the detection of IIA of cervical carcinoma was $80.0 \%, \quad 100.0 \%, \quad 100.0 \%, \quad 90.9 \%$ and $93.33 \%$ respectively. However, the sensitivity, specificity, PPV, NPV and accuracy of clinical examination of the detection of IIA of cervical carcinoma was $84.34 \%$, $63.86 \%, 84.87 \%, 45.86 \%$ and $67.44 \%$ respectively (Table 3).

Table 3: Diagnostic performance of MRI scan and Clinical staging for detection of carcinoma of cervix Stage IIA considering surgicohistopathological results as gold standard test

\begin{tabular}{lcc}
\hline Variables & MRI Values & Clinical Values \\
\hline Sensitivity & $80.0 \%$ & $84.34 \%$ \\
Specificity & $100.0 \%$ & $63.86 \%$ \\
PPV & $100.0 \%$ & $84.87 \%$ \\
NPV & $90.9 \%$ & $45.86 \%$ \\
Accuracy & $93.33 \%$ & $67.44 \%$ \\
\hline
\end{tabular}


The sensitivity, specificity, PPV, NPV and accuracy of MRI of the detection of IB of cervical carcinoma was $60.33 \%, \quad 55.36 \%, 64.5 \%, \quad 56.33 \%$ and $69.54 \%$ respectively. However, the sensitivity, specificity, PPV, NPV and accuracy of clinical examination of the detection of IB of cervical carcinoma were $0.0 \%$ in each (Table 4).

Table 4: Diagnostic Performance of MRI Scan and Clinical Staging for Detection of Carcinoma of Cervix Stage IIB Considering Surgico-Histopathological Results as Gold Standard Test

\begin{tabular}{lcc}
\hline Variables & MRI Values & Clinical Values \\
\hline Sensitivity & $60.33 \%$ & $0.0 \%$ \\
Specificity & $55.36 \%$ & $0.0 \%$ \\
PPV & $64.5 \%$ & $0.0 \%$ \\
NPV & $56.33 \%$ & $0.0 \%$ \\
Accuracy & $69.54 \%$ & $0.0 \%$ \\
\hline
\end{tabular}

The estimated area under the ROC curve was reported in both MRI and FIGO staging. MRI (0.86) was significantly better than FIGO staging (0.74) for staging cervical tumor in Stage IIA, as measured by the respective areas under the ROC curves (AUC) (Figure I).

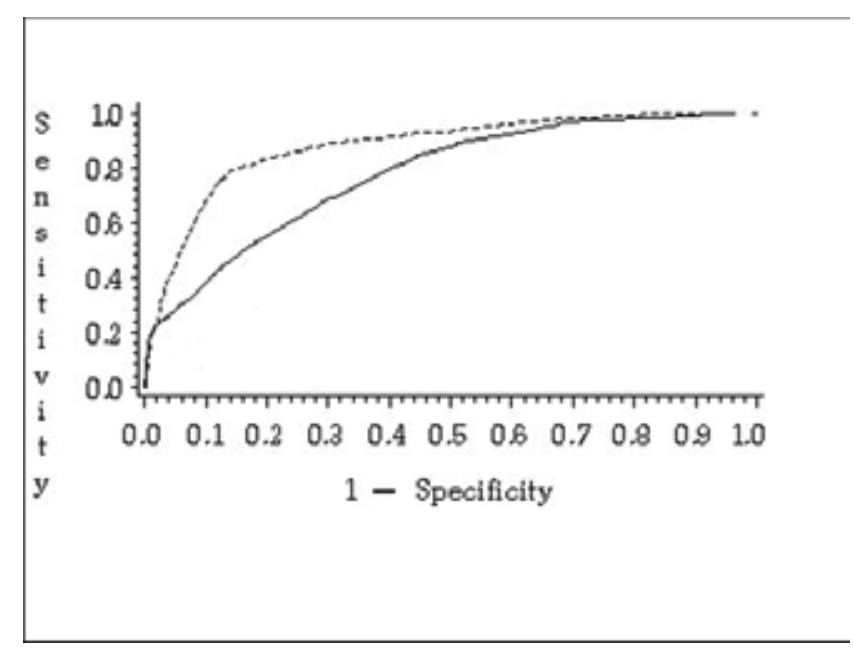

Figure I: Plots of receiver operating characteristic (ROC) curves for the assessment of stage IIA cervical carcinoma by magnetic resonance imaging $(\mathrm{MRI}=$ dotted line) and clinical $(\mathrm{FIGO}=$ black line $)$

\section{Discussion}

The diagnostic performance of MRI with FIGO clinical staging has been evaluated in the pretreatment of invasive cancer of the cervix in stage II using surgicohistopathological finding as reference standard. Hricak et $\mathrm{a}^{15}$ found invasion in $100.0 \%$ cases which was similar with the present study. The accurate staging of carcinoma of the cervix is important because the choice of treatment depends on the clinical stage of the disease at the time of initial examination. This cross sectional study has been carried out in the department of Radiology and Imaging at Dhaka Medical College Hospital, Dhaka, Bangladesh in collaboration with Obstetrics and Gynecology department from July 2010 to June 2012 to compare the levels of diagnostic performance of MRI with FIGO clinical staging in the pretreatment evaluation of invasive carcinoma of the cervix using surgicohistopathological findings as reference standard.

In present study, 10 had stage IIA and 6 had stage IIB. Regarding stage IIA, MRI diagnosed 8 patients and clinical examinations 20 patients in Stage IIA. Surgicopathologically stage IIB was confirmed in 6 cases, among them, four were correctly diagnosed by MRI, but clinical examination failed to stage any patient in this stage. These findings have been revealed that MRI staging is very much similar to surgicopathological staging and in this study FIGO staging could not detect any case beyond Stage IIA. In stage IIA, there is an overestimation by FIGO staging. This finding was similar with the study conducted by Hricak et a ${ }^{15}$. MRI image could demonstrate parametrium clearly. So, MRI staging gave better staging than FIGO staging. Sironi et $\mathrm{al}^{16}$ observed that overall accuracy of MR imaging in staging cervical carcinoma (stage IB-IIB) was $78.1 \%$. Ruben et $\mathrm{al}{ }^{17}$ have reported that MRI is seemed to be the most reliable preoperative modality for staging invasive cervical cancer. MR imaging should be used in conjunction with clinical staging to determine appropriate therapy in patients with stage IB -IIB cervical carcinoma.

For MRI, sensitivity, specificity, PPV, NPV and accuracy in IIA staging were $80.0 \%, 100.0 \%, 100.0 \%$, $90.9 \%$ and $93.33 \%$ respectively. FIGO IIA staging had sensitivity, specificity, PPV, NPV and accuracy $84.34 \%$, $63.86 \%, 84.87 \%, 45.86 \%$ and $67.44 \%$ respectively. It was observed that clinical staging was possible up to IIA stage. Therefore, beyond IIA stage diagnostic accuracy was not possible to calculate. In Stage IIB, sensitivity, specificity, PPV, NPV and accuracy were $60.33 \%$, $55.36 \%, 64.5 \%, 56.33 \%$ and $69.54 \%$ respectively for MRI scan.

For each modality (FIGO and MRI scan) in present study, the estimated area under the ROC curve was reported. In case of Stage IIA, MRI (0.86) was also 
proved to be significantly better than FIGO staging (0.74) for staging cervical tumor. These results revealed that MRI staging was more accurate than FIGO staging in diagnosis of invasive cervical carcinoma. Rockall et al ${ }^{18}$ has defined Stage IA as a microinvasive tumor that cannot be reliably shown on T2-weighted images. However, microinvasive disease might be detected on dynamic MRI as a strongly enhancing area on early arterial phase images. MRI is more accurate for predicting macroinvasive tumor and in showing its relationship of to the internals and surrounding structures.

This could potentially lead to a reduction in staging cost and morbidity. In patients with endometrial and cervical cancer, the presence of lymph node metastasis suggested a poor prognosis, with a marked decrease in survival rate. Lymph node involvement which was not included in FIGO staging system of carcinoma of cervix was also an important factor in choice of adjuvant radiation therapy in both endometrial and cervical cancer.

The estimated area under the ROC curve was reported in both MRI and FIGO staging. MRI (0.86) was significantly better than FIGO staging $(0.74)$ for staging cervical tumor in Stage IIA, as measured by the respective areas under the ROC curves (AUC). From the results of the present study and the findings obtained by others, it is conceivable that MR imaging is a reliable imaging technique for diagnosing, staging and also evaluating important prognostic factors of carcinoma of cervix stage IIA. Images of the pelvis, as compared with those of abdomen are better quality because respiratory and intestinal motion are minimum. However, MRI also has certain limitations. These include a relatively long scanning time and the contraindication to scan patients with cardiac pace makers, intracranial vascular clips and large metallic devices.

\section{Conclusion}

In conclusion most common age group of cervical carcinoma is in the old age. However, the detection capacity of MRI is higher than clinical examination among cervical carcinoma patients in stage II patients. Furthermore the comparison of ROC curve shows that the value of AUC of MRI is more than the AUC value of clinical examination among women presented with stage II cervical carcinoma. However, a large scale study should be conducted.

\section{References}

1. Nicolet V, Carignan L, Bourdon F, Prosmanne O. MR imaging of cervical carcinoma: a practical staging approach. Radiographics. 2000;20(6):1539-49

2. Mahajan M, Kuber R, Chaudhari KR, Chaudhari P, Ghadage P, Naik R. MR imaging of carcinoma cervix. The Indian journal of radiology \& imaging. 2013;23(3):247

3. Boss EA, Barentsz JO, Massuger LF, Boonstra H. The role of MR imaging in invasive cervical carcinoma. European radiology. 2000;10(2):256-70

4. World Health Organization. Fact sheet No.297:Cancer, 2006

5. Zand KR, Reinhold C, Abe H, Maheshwari S, Mohamed A, Upegui D. Magnetic resonance imaging of the cervix. Cancer Imaging. 2007;7(1):69

6. Okamoto Y, Tanaka YO, Nishida M, Tsunoda H, Yoshikawa H, Itai Y. MR imaging of the uterine cervix: imaging-pathologic correlation. Radiographics. 2003;23(2):425-45

7. Szklaruk J, Tamm EP, Choi H, Varavithya V. MR imaging of common and uncommon large pelvic masses. Radiographics. 2003;23(2):403-24

8. Pecorelli S. Revised FIGO staging for carcinoma of the vulva, cervix, and endometrium. International Journal of Gynecology \& Obstetrics. 2009;105(2):103-4

9. Kaur H, Silverman PM, Iyer RB, Verschraegen CF, Eifel PJ, Charnsangavej C. Diagnosis, staging, and surveillance of cervical carcinoma. American Journal of Roentgenology. 2003;180(6):1621-31

10. Engin G, Küçücük S, Ölmez H, Hasiloglu ZI, Disçi R, Aslay I. Correlation of clinical and MRI staging in cervical carcinoma treated with radiation therapy: a single-center experience. Diagnostic and Interventional Radiology. 2011;17(1):44

11. Szklaruk J, Tamm EP, Choi H, Varavithya V. MR imaging of common and uncommon large pelvic masses. Radiographics. 2003;23(2):403-24

12. Chung HH, Kang SB, Cho JY, Kim JW, Park NH, Song YS, Kim $\mathrm{SH}$, Lee HP. Can preoperative MRI accurately evaluate nodal and parametrial invasion in early stage cervical cancer?. Japanese journal of clinical oncology. 2007;37(5):370-5

13. Follen M, Levenback CF, Iyer RB, Grigsby PW, Boss EA, Delpassand ES, Fornage BD, Fishman EK. Imaging in cervical cancer. Cancer: Interdisciplinary International Journal of the American Cancer Society. 2003 Nov 1;98(S9):2028-38

14. Sala E, Wakely S, Senior E, Lomas D. MRI of malignant neoplasms of the uterine corpus and cervix. American Journal of Roentgenology. 2007;188(6):1577-87

15. Hricak H, Lacey CG, Sandles LG, Chang YC, Winkler ML, Stern JL. Invasive cervical carcinoma: comparison of MR imaging and surgical findings. Radiology. 1988;166(3):623-31

16. Sironi S, Belloni C, Taccagni GL, DelMaschio A. Carcinoma of the cervix: value of MR imaging in detecting parametrial involvement. AJR. American journal of roentgenology. 1991;156(4):753-6

17. Rubens D, Thornbury JR, Angel C, Stoler MH, Weiss SL, Lerner RM, Beecham J. Stage IB cervical carcinoma: comparison of clinical, MR, and pathologic staging. American Journal of Roentgenology. 1988;150(1):135-8

18. Rockall AG, Ghosh S, Alexander-Sefre F, Babar S, Younis MT, Naz S, Jacobs IJ, Reznek RH. Can MRI rule out bladder and rectal invasion in cervical cancer to help select patients for limited EUA? Gynecologic oncology. 2006;101(2):244-9 